\title{
Facilitators and barriers to self-management of nursing home residents: perspectives of health- care professionals in Korean nursing homes
}

This article was published in the following Dove Press journal:

Clinical Interventions in Aging

12 October 2015

Number of times this article has been viewed

\author{
Yeon-Hwan Park ${ }^{1,2}$ \\ Hwal Lan Bang ${ }^{2,3}$ \\ Ga Hye Kim' \\ Ji Yeon $\mathrm{Ha}^{\prime}$
}

'College of Nursing, Seoul National University, Seoul, Korea; ${ }^{2}$ The

Research Institute of Nursing

Science, Seoul National University,

Seoul, Korea; ${ }^{3}$ Department of

Nursing Science, College of Industry,

Sangmyung University, Cheonan, Korea

Correspondence: Hwal Lan Bang

Department of Nursing Science, College

of Industry, Sangmyung University,

3I Sangmyungdae-gil, Dongnam-gu,

Cheonan, 31066, Korea

$\mathrm{Tel}+82415505494$

Fax +82 4I 5505545

Email luvlucie@smu.ac.kr
Purpose: To explore facilitators and barriers to self-management from the viewpoint of staff taking care of nursing home (NH) residents with chronic diseases in South Korea.

Patients and methods: A qualitative content analysis was done using the focus group interview method. A total of 23 health-care professionals ( 16 registered nurses and 7 social workers) were interviewed from three urban NHs, each with more than 100 beds.

Results: Five facilitators were identified: grouping the residents; the resident's awareness of his/her current health status; the willingness of residents to engage in self-management; residence in the facility; and support from the staff. Additionally, seven barriers were identified: deterioration of the resident's health; the dependency expectations of the resident; hesitation in asking for help; difference in expectations between the staff and the resident's family; insufficient staffing and time; lack of standardized guidelines; and conservative tendencies of the staff due to rigid policies.

Conclusion: The findings of this study can help health-care professionals recognize the factors that influence self-management and provide direction for registered nurses and other health professionals involved in supporting self-management programs for $\mathrm{NH}$ residents.

Keywords: self-management, aged, focus groups, long-term care

\section{Introduction}

With the growing number of older people suffering from chronic illnesses, the demand for long-term care is increasing worldwide. ${ }^{1}$ The percentage of older adults with chronic illnesses in Korea has been estimated to be as high as $88.5 \%$, with an average of 2.5 chronic diseases affecting community-dwelling older adults and 3.23 chronic diseases on average among nursing home (NH) residents. ${ }^{2}$ Self-management can be defined as the decisions and behaviors that subjects with chronic illness engage in that affect their health. ${ }^{3}$ It is pivotal in patients with chronic diseases because they can manage their symptoms, treatment, and lifestyle changes inherent when living with a chronic condition, ${ }^{4}$ and most of the health problems related to chronic illness can be prevented by self-management..$^{5}$ The need for self-management programs for NH residents is increasing, ${ }^{6}$ although not without debate. Some suggest that self-management has no place in NHs and is often limited to long-term care facilities because dependency is primarily seen as a result of significant physical and cognitive decline of the residents, ${ }^{7}$ and suffering from major chronic illnesses can add to their feeling of dependence. ${ }^{8}$ In a recent study, $92.7 \%$ of the Korean $\mathrm{NH}$ residents were found to be functionally dependent and 90.8\% were cognitively impaired. ${ }^{9}$ Nevertheless, others have reported that $\mathrm{NH}$ residents still want to be in control of their care. ${ }^{6,10}$ Given these divergent 
opinions, understanding the facilitating or disturbing factors related to self-management is critical for developing effective programs. ${ }^{11}$ Most of the studies regarding these factors were conducted from the perspective of the patients ${ }^{12}$ with specific chronic diseases such as diabetes ${ }^{13-15}$ in settings other than NHs. Furthermore, most of these studies have been reported in Western countries. ${ }^{13}$ The older adults in a unique sociocultural context such as that of South Korea may experience a different set of circumstances than in Western countries. As a result, little is known about self-management among the older adults living in NHs in South Korea. Deeply rooted in Confucianism, Korean society demonstrates a strong sense of elder respect. ${ }^{16}$ The "care respect" is expressed by providing personal care, nourishment, and health and social services for elders. ${ }^{16}$ It is somewhat natural for Korean senior adults to be dependent on caregivers, but it might interfere with self-management. Cultural context aside, NH residents experience contradictory feelings such as sorrow and relief when entering into a dependent life in a public environment such as a NH. ${ }^{17}$ Health-care professionals should initiate or arrange adequate care during the care trajectory ${ }^{18}$ and support residents' self-management in any setting, including NHs. If residents were more involved in self-management, they would show better health behaviors and improvement in the control of chronic diseases. ${ }^{18}$ An understanding of the barriers and facilitators will allow health-care professionals to better assist residents in the self-management of their chronic health status, which requires numerous daily decisions. ${ }^{19}$

The purpose of this study was to explore barriers and facilitators to self-management of $\mathrm{NH}$ residents with chronic diseases from the perspective of health-care professionals in South Korea. On the basis of the findings, health-care professionals could support the self-management of $\mathrm{NH}$ residents by developing culturally relevant and practical self-management programs in the future.

\section{Methods}

\section{Setting and participants}

The participants were recruited by purposive sampling. The inclusion criteria were the following: 1) health-care professionals currently employed in NHs, 2) at least 1 year of work experience in NHs. To recruit potential participants for focus groups (FGs), the authors contacted three NHs selected on a convenience basis for their accessibility from the list of NHs within Seoul with more than 100 beds. The managers in NHs recruited the staff according to the inclusion criteria, reassuring the eligible participants that participation in this study is voluntary and refusal to participate will involve no penalty.
The participating health-care professionals were mainly registered nurses (RNs) and social workers. Consequently, in this study, a total of 16 RNs and 7 social workers participated. This study was approved by the Institutional Review Board of Seoul National University prior to data collection. The participants were told that participation was voluntary and that they could withdraw from the study at any time. They were also told that they could ask for any data collected to be excluded from analysis if they had any concerns. Consent forms were signed by all participants.

\section{Data collection}

Data were collected from the FG interviews. There were three FGs: six RNs and five social workers were recruited from a NH in Seoul (group 1); four RNs and two social workers were recruited from another urban NH (group 2), and six RNs were recruited from another NH (group 3). All NHs had more than 100 beds.

The FGs were conducted one at a time until saturation was reached and no new themes emerged. The data collection was completed after the interview with the third FG.

An experienced moderator (YHP) moderated the sessions, guided by semistructured questions (listed in Table 1). Open-ended questions, combined with a series of probing questions, were designed to elicit barriers and facilitators to self-management of NH residents from the perspective of health-care professionals. To enhance the reliability of the data collection, the same moderator moderated all three FGs. For quality assurance, two facilitators (HLB and GHK) were present during the interview of FGs to take field notes including nonverbal gestures and expressions. The groups began with a brief introduction of the study and general instructions that stated that the participants were allowed to freely express their experiences, opinions, and feelings. Each session took 60-90 minutes. For clarification and verification, the main points of the discussion were summarized by the moderator before completing the session. All sessions were audio-recorded and transcribed. Immediately after the interview with each FG, the moderator and facilitators met for debriefing on the data and to get their overall impressions from the FG interview. These discussions were used iteratively to inform subsequent group interviews and to detect potential gaps in the content.

\section{Data analysis}

Qualitative content analysis was used to analyze the data from the FG interviews. The analysis consisted of a preparation, an organization, and a result phase. ${ }^{20}$ In the preparation 
Table I Focus group interview guide

\section{Introductory question}

- Please tell us about your general and specific experiences in the management of chronic health disorders of $\mathrm{NH}$ residents.

Transition questions

- How much do the residents suffer from their chronic illnesses?

- To what extent do the residents perform their self-management?

Key questions

- What are the tasks or activities that help the residents manage their own health in the $\mathrm{NH}$ ?

- What are the factors that interfere with the residents' managing (ie, medication, nutrition, physical activity, sleep, stress management, and/or compliance to therapeutic measures) their own chronic health problems?

- What are the factors that help the residents manage (ie, medication, nutrition, physical activity, sleep, stress management, and/or compliance to therapeutic measures) their own chronic health problems?

Ending question

- We have talked about the barriers and facilitators to self-management of the residents so far, please feel free to add anything that you think is necessary in order to improve their self-management.

Abbreviation: $\mathrm{NH}$, nursing home.

phase, all of the FG transcripts were selected as the unit of analysis. ${ }^{21}$ Two authors read each transcript independently several times to make sense of the data and to obtain a sense of the whole. In the organization phase, meaningful units were underlined and condensed. The condensed meaningful units were abstracted and labeled with a code. The various codes were compared based on the differences and similarities and then sorted into two categories: barriers and facilitators. Identified themes were reviewed and discussed during weekly meetings with team members, and an independent expert in qualitative research reviewed the themes for verification and refinement.

\section{Results}

The 23 health-care professionals participating in this study included 21 females and 2 males, with an age range of 29-58 years (45.9 \pm 8.9$)$ and experience ranging from 6 to 108 months (51.5 \pm 35.6$)$. We focused on the broad themes of barriers and facilitators to self-management from the perspective of health-care professionals working in NHs and identified five facilitators and seven barriers, summarized in Table 2. The findings are listed in rank order from the most prevalent themes to the least prevalent.

\section{Facilitators to self-management}

The perceived facilitators to self-management of NH residents are listed in the order of frequency from the most discussed to those least discussed by the participants.

\section{Grouping the residents}

The health-care professionals mentioned that if the residents were grouped according to their cognitive level or functioning capacity, it would be easier for them to support the residents with individualized care needs and to help them be engaged in self-management activities.

[...] it' $\mathrm{d}$ be possible if the seniors of similar functional level are gathered together. You provide intense care to more severe conditions[...]. I think if they can maintain 'self-care' to a certain level, self-management might be achievable. [FG2, RN3]

Each resident has their own needs, and these are so diverse. We grouped these needs - for example, exercise, music, massage and so on. Last year, it was quite successful for us to motivate their participation in self-management. [FG1, RN1]

\section{The resident's awareness of current health status}

Participants discussed that if the residents were aware of their health status and what they needed to do, then getting involved in self-management activities might be easier.

Table 2 Facilitators and barriers to self-management in $\mathrm{NHs}$

\section{Facilitators}

Grouping the residents.

The resident's awareness of his/her current health status.

Willingness of the resident to engage in self-management.

Residence in the facility.

Support from the staff.

\section{Barriers}

Deterioration of the resident's health.

Dependency expectations of the resident.

Hesitation in asking for help.

Difference in expectations (between the staff and the resident's family). Insufficient staffing and time.

Lack of standardized guidelines.

Conservative tendencies of the staff due to rigid policies.

Abbreviation: $\mathrm{NH}$, nursing home. 
Seniors with intact cognition refrain from snacking on their own if they are aware that they gained weight. [FG1, RN2]

After telling them the reason to be clean, if they know the necessity and why they need to do it, they do it by themselves if they can. [FG1, RN1]

\section{Willingness of the resident to engage in self-management}

Many participants agreed that if the resident is motivated and he or she initiates the self-care, the self-management may be sustained steadily.

She began by herself, practices like writing and line drawing at first. She asked for it. She planned it herself, saying 'I'm doing this much today' [...] [FG3, RN4]

$[\ldots]$ although some assistance is needed for moving, it (self-management) is possible if they can manage their own time schedule, for instance, 'I'm doing so and so on this time slot' $[\ldots][\mathrm{FG} 1, \mathrm{RN} 1]$

\section{Residence in the facility}

Although not as widely discussed compared to the above factors, some participants cited the location itself as an additional facilitator to self-management. Living in an $\mathrm{NH}$ itself increases the self-management behavior of the resident.

It's better to live in the facility and to utilize our help than staying at home. It is spacious here. They tend to move around more. It helps them to maintain functional ability to some extent. [...] Once they come here, they move here and there to keep up with the group living, whether they want it or not. Their mobility is increased sufficiently either by using wheelchairs or other's help. [FG1, SW2]

Sometimes, residents seem to do things by themselves that they would not have done while at home [alone]. [FG3, RN2]

\section{Support from the staff}

Constant support from the staff and ongoing education help residents engage in self-management activities.

Keep encouraging seniors consistently, because they can do it. [...]make them do it, although not perfect, holding spoons by themselves. Keep offering such stimuli. [FG1, SW1]

I think it's the right thing to keep on telling them this, even when they do not seem to understand it. [FG2, RN5]

\section{Barriers to self-management}

FG participants reported multiple barriers to self-management of $\mathrm{NH}$ residents. We did not observe distinct differences in perceived barriers by sex, profession, or NH. The most frequently reported barriers are as follows.

\section{Deterioration of the resident's health}

The most common barrier mentioned by participants was the deterioration of health status of the resident. Worsening chronic diseases inevitably interfere with self-management, even if the resident has intention to care for themselves on their own.

For instance, if a senior suffers from Parkinsonism, their physical health gradually deteriorates. Then, it is impossible for them to manage themselves, even though they are willing to do so. [FG1, RN1]

Once their cognitive function gets worse, selfmanagement is almost impossible even though their mobility is not limited. [FG1, RN2]

\section{Dependency expectations of the resident}

Although dependency can be expected as being part of elder respect in many Asian cultures, it was mentioned in each FG interview as a barrier to self-management.

Once they enter our nursing home, the residents and their family members expect us to do everything for them just because they are seniors. [FG2, RN4]

There was a senior who could eat by himself. But when we helped another senior with feeding, the first senior put down his spoon, waiting for us to help him. Once you help them, it never ends. It seems that they have a firm belief that they have the right to be assisted, that is, to be dependent. [FG2, RN6]

\section{Hesitation in asking for help}

While general dependency can interfere with self-management, residents do need varying degrees of assistance from the staff to be engaged actively in self-management activities. However, in Asian cultures, especially Korea's, people hesitate to ask others for help because they perceive it as causing trouble for others. Due to this hesitation, residents might lose opportunities for self-management because they are reluctant to request for basic assistance that they deserve.

They don't easily request for assistance even when they need something because they are sorry to ask for help. It might worsen their health when they keep lying down so long. [FG1, RN2] 
They are hesitant in asking for help because they don't want to trouble others for their inconvenience. They'd rather choose to refuse participating in activity than to ask for help. Eventually, we tend to exclude them afterwards. [FG1, SW2]

\section{Difference in expectations (between the staff and the resident's family)}

If the resident's family members do not have the same idea about what the resident can do by him or herself, then the staff's decision for self-management activities might not be appreciated by the family. Some family members view the staff's promotion of self-management as abandonment, or some view it as excessive and unnecessary.

When the seniors eat by themselves and use the toilet independently, their daughters or sons feel bad for their parents. They consider they are treated properly only when the staffs do everything for them. We let them eat by themselves with their residual function even though it's messy. But the families accuse us 'what are the staffs doing?' when they witness the messy scene. [FG2, RN3]

Some family members don't want us to do any more for the residents. 'Why bother? What else can they do?' They say $[\ldots]$ They seem to think that we are fussing over too much with the residents' self-management'. [FG3, RN2]

\section{Insufficient staffing and time}

Participants voiced strongly that if they had enough time and personnel, they could offer sufficient time and room for the residents for self-care and self-management activities.

The problem is, you know, the staffing. If there were sufficient staffs, we could afford the time to let them attempt self-care on their own and observe. But, you see, when workload is high, you tend to do things for them quickly in order to turn to your next resident. [FG2, RN1]

If there were enough time, we could wait and let them eat by themselves and let them take their medication on their own. But time is limited. [FG3, RN3]

\section{Lack of standardized guidelines}

Participants specified that the direction of the care is not always agreed upon among the staff because there are no guidelines for supporting the residents with selfmanagement.

It's quite different according to care teams. One team decides to give seniors a chance, while another team doesn't. [FG2, RN5]
RNs and NAs do not go together. It just doesn't work. If only there were some guidelines [...] [FG3, RN1]

\section{Conservative tendencies of the staff due to rigid policies}

Policies designed to place safety as the top priority may restrict the staff's view of what is permitted as self-management behaviors like self-medication. The health-care providers, for example, oftentimes cannot help but restrict the residents from holding their medication on their own because of liability.

We tend to be rather defensive. Loading too much responsibility on staffs might lower the self-management ability of the seniors. [FG1, RN1]

To conform to the criteria for Health Insurance audit rating, we cannot permit the residents to self-medicate or possess any medication, not even any health food. [FG3, RN1]

\section{Discussion}

To our knowledge, this is the first study to identify the facilitators and barriers to self-management in NH residents as perceived by health-care professionals in South Korea. Our key findings were as follows: facilitators include grouping the residents; the resident's awareness of his/her current health status; willingness of the resident to engage in selfmanagement; residence in the facility; and support from the staff. The barriers include deterioration of the resident's health, dependency expectations of the resident, hesitation in asking for help, difference in expectations between the staff and the resident's family, insufficient staffing and time, lack of standardized guidelines, and conservative tendencies of the staff due to rigid policies.

Most of the previous studies have focused on the views of the older adults on patient involvement or participation, either in primary care, ${ }^{22}$ in care during hospitalization, ${ }^{23}$ or when being assessed for receiving public home help. ${ }^{24}$ The concept of self-management has been established in relation to how subjects can gain and remain in control of their care process and how professionals can support this process. ${ }^{3}$ In our study, from the viewpoint of health-care professionals, grouping the residents emerged as the major facilitator to self-management of $\mathrm{NH}$ residents. The health-care professionals suggested that it would be more efficient to cater to groups of elders with a similar physical and cognitive status. However, the desire for involvement in self-management may be highly heterogeneous, ${ }^{22}$ so an individualized approach 
for each resident is needed. Residence in the facility was another facilitator to self-management. Living in and needing care in a public environment such as an $\mathrm{NH}$ could be a new opportunity or challenge for older people to change their life styles. ${ }^{17}$ However, life quality in an institutionalized environment such as $\mathrm{NH}$ is often regarded as being strongly compromised. ${ }^{25}$ Abilities and environmental resources for engaging in health behaviors were reported to be more restricted for $\mathrm{NH}$ residents than community-dwelling older adults. ${ }^{26} \mathrm{NH}$ residents noted that when staff "took care of things," their freedom for independent choice was reduced; however, some residents emphasized how their functionality had improved since living in the $\mathrm{NH}^{25}$ Older adults, especially those who had been hospitalized before, regarded life in an NH to be more active with more opportunities for personal independence. ${ }^{25}$ Living in an $\mathrm{NH}$, where fellow residents form a community and professional assistance is readily available, is more conducive to self-management than staying at home, where the resident is relatively isolated. It was reported that easy access to care is important in older adult's involvement in their own care. ${ }^{22}$ A similar facilitator is "support from the staff." Many studies reported that health professionals influence the self-management behavior of the clients. ${ }^{13,27}$ Our findings are consistent with those from previous studies that reported that the availability and accessibility of the health professional were essential to a self-management program, ${ }^{22}$ and the support from health-care professionals led to the facilitation of effective self-management. ${ }^{6}$

Facilitators from this study reflect the major characteristics of self-management. In general, self-management is a dynamic and continuous process that refers to the individual's ability to manage his/her symptoms, treatment, physical and psychosocial consequences, and life style changes inherent when living with a chronic condition. ${ }^{4}$ The results of this study are consistent with and extend those of previous studies in that NH residents still want to be in control of their own care. ${ }^{10}$ The frail older adults are increasingly encouraged to be in control of their health care, especially in Western societies. ${ }^{18} \mathrm{NH}$ residents with low functional level were often believed to have no such capacity, but functional independence may allow them to follow self-management strategies. ${ }^{6}$ Although not all older people residing in NHs have the condition required to participate in self-management, being aware of the importance of self-management and being willing to control their health and life could be essential factors in facilitating their self-management in NH. Therefore, it is necessary for the health-care professionals to continuously support and motivate $\mathrm{NH}$ residents in order for them to maintain their independence and to participate actively in various self-management behaviors.

It is like two sides of the same coin in that some barriers were exactly the opposite of the facilitators. The deterioration of health status of a resident was the first and most commonly mentioned barrier to self-management in a $\mathrm{NH}$. Because "grouping the residents" was the major facilitator to self-management in this study, we could easily assume that it might be desirable to exclude those with deteriorating health status from a self-management program by "grouping the residents" according to their cognitive level or functional capacity. However, with the high prevalence of major chronic diseases (in demand of self-management such as hypertension) among $\mathrm{NH}$ residents, ${ }^{28}$ it will be necessary to develop and implement effective self-management programs that help the residents - even those with the deteriorating health status - maintain their health and quality of life. "Dependency expectations of the resident," "hesitation in asking for help," and "difference in expectations between the staff and the resident's family" are the barriers that might be due to Asian, especially Korean, culture. Generally, older Korean adults and their families think that NHs are not so different from clinical settings like geriatric hospitals, and they actually choose to admit them to either of these facilities interchangeably. ${ }^{29}$ They tend to be dependent on and rely on staff in an $\mathrm{NH}$, even with whatever they can manage by themselves, ${ }^{29}$ as if they were helpless. Additionally, under the strong influence of Confucianism, filial piety is very important in Korea. ${ }^{16}$ In turn, the offspring of the residents may feel guilty for admitting their parents to NHs and ostensibly failing to take care of their own parents. ${ }^{30}$ We can assume that they expect NH staff to provide total care with respect and do "everything" for their parents instead of providing opportunities for self-management. In contrast, some family members do not want any more active plans or programs to be done for their parents or spouses because they do not think that there still is hope for improvement or self-management of the resident. It is necessary to communicate with such family members about the importance of self-management with staff support. It is important that the residents be involved in selfmanagement through a collaborative relationship between the health professionals and residents and their families. ${ }^{3}$

Being dependent but being hesitant to ask for help at the same time is somewhat ironic. It is another aspect of Korean culture that they are dependent, but they still want to be courteous and polite to others at the same time. Instead of initiating any active self-management by asking for some help, they choose to be courteous and be a "good" resident. 
The health-care professionals need to encourage the residents and the family to participate in self-management from the time the residents enter the $\mathrm{NH}$ and thereafter from time to time. It is also necessary to tell them how much the resident can do to manage themselves and to what extent care and services are provided.

The results of this study highlight the need for more policy changes. One of the important barriers was fewer qualified health-care professionals. Previous studies have shown that an adequate number of well-trained and stable nursing staff is essential for providing adequate quality care in long-term care settings. ${ }^{31}$ Without the employer dictating a certain residentto-staff ratio, it would not be possible to provide high-quality service, including self-management program. Additionally, each NH service should be adequately monitored and accredited for services after they meet certain standards. Continuing education needs to be provided and required for $\mathrm{NH}$ staff to become educated in standardized guidelines. Additionally, the results of this study showed that NH staff perceive many dilemmas between the key contents of self-management programs and the rigid NH regulations. Studies have found that role conflicts limit the ability of RNs to provide adequate care at long-term care settings. ${ }^{31,32}$ Flexible coordination of services based on the needs and health status of $\mathrm{NH}$ residents needs to be the main goal of NHs.

A limitation of this study is a possible selection bias. Because we interviewed those who voluntarily chose to participate in the study, these participants could have been more interested in self-management. The limitations of FG studies are well recognized. In particular, the fact that the researcher directs the group and group dynamics influence which participants contribute and what they contribute could bias the results. However, FG methodology that was moderated by an experienced researcher and guided by semistructured questions allowed us to bring together insights into health professionals' perceptions of facilitators and barriers to self-management. In this study, both RNs and social workers were interviewed together as a group, and this might influence the findings by limiting the willingness of participants to reveal beliefs and feelings in the presence of other professionals.

\section{Conclusion}

In conclusion, our study identified a variety of facilitators and barriers to self-management of residents in NHs from the perspective of health-care professionals. From the view of the health-care professionals, grouping the residents according to their physical and cognitive capabilities was particularly important in facilitating the resident's selfmanagement. The major barrier to self-management was the deterioration of the resident's health. This implies that a self-management program needs to be tailored specifically to the resident's health status, which requires enough staffing and time with standardized guidelines. In addition to the residents' awareness and willingness for self-management, continuous support from the staff is desirable. The findings from this study should help RNs recognize the factors that influence self-management and provide direction for RNs and other health professionals involved in supporting selfmanagement programs for NH residents. Future studies should find and develop effective practices that exploit the facilitators identified while at the same time address the barriers to self-management $\mathrm{NH}$ residents.

\section{Acknowledgment}

This study was supported by Basic Science Research Program through the National Research Foundation of Korea (NRF) funded by the Ministry of Education, Science and Technology (MEST), (grant number 2013R1A1A3A04008055).

\section{Disclosure}

The authors report no conflicts of interest in this work.

\section{References}

1. Sevick MA, Trauth JM, Ling BS, et al. Patients with complex chronic diseases: perspectives on supporting self-management. J Gen Intern Med. 2007;22(Suppl 3):438-444.

2. Korea Institute for Health and Social Affairs. Survey of the Elderly 2011; 2011. Available from: https://www.kihasa.re.kr/html/jsp/publication/ policy/view.jsp?bid=12\&ano=1338. Accessed July 27, 2014.

3. Johnston B, Rogerson L, Macijauskiene J, Bla A, Cholewka P. An exploration of self-management support in the context of palliative nursing: a modified concept analysis. BMC Nurs. 2014;13(1):21.

4. Barlow J, Wright C, Sheasby J, Turner A, Hainsworth J. Self-management approaches for people with chronic conditions: a review. Patient Educ Couns. 2002;48(2):177-187.

5. Jordan JE, Briggs AM, Brand CA, Osborne RH. Enhancing patient engagement in chronic disease self-management support initiatives in Australia: the need for an integrated approach. Med J Aust. 2008; 189(10 Suppl):S9-S13.

6. Park YH, Chang H, Kim J, Kwak JS. Patient-tailored self-management intervention for older adults with hypertension in a nursing home J Clin Nurs. 2013;22(5-6):710-722.

7. Baltes MM. The etiology and maintenance of dependency in the elderly: three phases of operant research. Behav Ther. 1988;19(3):301-319.

8. Stabell A, Eide H, Solheim GA, Solberg KN, Rustøen T. Nursing home residents' dependence and independence. J Clin Nurs. 2004; 13(6):677-686.

9. Kim JI. Prediction of quality of life among the elderly at care facilities for the elderly according to health states, physical and cognitive functions, and social supports-focused on D metropolitan city. J Korea Acad-Ind Coop Soc. 2015;16(7):4656-4667.

10. Strohbuecker B, Eisenmann Y, Galushko M, Montag T, Voltz R. Palliative care needs of chronically ill nursing home residents in Germany: focusing on living, not dying. Int J Palliat Nurs. 2011;17(1):27. 
11. Wilson C, Alam R, Latif S, Knighting K, Williamson S, Beaver K. Patient access to healthcare services and optimisation of self-management for ethnic minority populations living with diabetes: a systematic review. Health Soc Care Community. 2012;20(1):1-19.

12. Jerant AF, Friederichs-Fitzwater MM, Moore M. Patients' perceived barriers to active self-management of chronic conditions. Patient Educ Couns. 2005;57(3):300-307.

13. Shen H, Edwards H, Courtney M, McDowell J, Wei J. Barriers and facilitators to diabetes self-management: perspectives of older community dwellers and health professionals in China. Int $J$ Nurs Pract. 2013;19(6):627-635.

14. Song M, Lee M, Shim B. Barriers to and facilitators of self-management adherence in Korean older adults with type 2 diabetes. Int $J$ Older People Nurs. 2010;5(3):211-218.

15. Choi S, Song M, Chang SJ, Kim S-A. Strategies for enhancing information, motivation, and skills for self-management behavior changes: a qualitative study of diabetes care for older adults in Korea. Patient Prefer Adherence. 2014;8:219-226.

16. Sung K-t. Elder respect: exploration of ideals and forms in East Asia. J Aging Stud. 2001;15(1):13-26.

17. Anderberg P, Berglund A-L. Elderly persons' experiences of striving to receive care on their own terms in nursing homes. Int J Nurs Pract. 2010;16(1):64-68.

18. Claassens L, Widdershoven G, Van Rhijn S, et al. Perceived control in health care: a conceptual model based on experiences of frail older adults. J Aging Stud. 2014;31:159-170.

19. Nagelkerk J, Reick K, Meengs L. Perceived barriers and effective strategies to diabetes self-management. J Adv Nurs. 2006;54(2): $151-158$.

20. Elo S, Kyngas $\mathrm{H}$. The qualitative content analysis process. $J$ Adv Nurs. 2008;62(1):107-115.

21. Graneheim UH, Lundman B. Qualitative content analysis in nursing research: concepts, procedures and measures to achieve trustworthiness. Nurse Educ Today. 2004;24(2):105-112.
22. Bastiaens H, Van Royen P, Pavlic DR, Raposo V, Baker R. Older people's preferences for involvement in their own care: a qualitative study in primary health care in 11 European countries. Patient Educ Couns. 2007; 68(1):33-42.

23. Ekdahl AW, Andersson L, Friedrichsen M. "They do what they think is the best for me." Frail elderly patients' preferences for participation in their care during hospitalization. Patient Educ Couns. 2010;80(2):233-240.

24. Janlöv A-C, Hallberg IR, Petersson K. Older persons' experience of being assessed for and receiving public home help: do they have any influence over it? Health Soc Care Community. 2006;14(1):26-36.

25. Stathi A, Simey P. Quality of life in the fourth age: exercise experiences of nursing home residents. J Aging Phys Act. 2007;15(3):272-286.

26. Chen Y-M, Li Y-P. Motivators for physical activity among ambulatory nursing home older residents. Sci World J. 2014;2014:329397.

27. Bair MJ, Matthias MS, Nyland KA, et al. Barriers and facilitators to chronic pain self-management: a qualitative study of primary care patients with comorbid musculoskeletal pain and depression. Pain Med. 2009;10(7):1280-1290.

28. Moore K, Boscardin W, Steinman M, Schwartz JB. Patterns of chronic co-morbid medical conditions in older residents of US nursing homes: differences between the sexes and across the agespan. J Nutr Health Aging. 2014;18(4):429-436.

29. Kim JS, Sunwoo D, Lee KJ, Choi ID, Lee HY, Kim KA. Establishing Role of Nursing Home and Geriatric Hospital: Reinforcing Medical Service Linkage. Seoul, South Korea: Korea Institute for Health and Social Affairs (KIHASA); 2013.

30. Kwon S, Tae Y-S. The experience of adult Korean children caring for parents institutionalized with dementia. J Korean Acad Nurs. 2014; 44(1):41-54.

31. Dabelko HI, Zimmerman JA. Outcomes of adult day services for participants: a conceptual model. J Appl Gerontol. 2008;27(1):78-92.

32. Bull MJ, McShane RE. Seeking what's best during the transition to adult day health services. Qual Health Res. 2008;18(5):597-605.
Clinical Interventions in Aging

\section{Publish your work in this journal}

Clinical Interventions in Aging is an international, peer-reviewed journal focusing on evidence-based reports on the value or lack thereof of treatments intended to prevent or delay the onset of maladaptive correlates of aging in human beings. This journal is indexed on PubMed Central, MedLine,

\section{Dovepress}

CAS, Scopus and the Elsevier Bibliographic databases. The manuscript management system is completely online and includes a very quick and fair peer-review system, which is all easy to use. Visit http://www.dovepress. com/testimonials.php to read real quotes from published authors. 